\title{
LETTER \\ Stacked Rectangular Microstrip Antenna with a Shorting Plate for Dual Band (VICS/ETC) Operation in ITS
}

\author{
Takafumi FUJIMOTO $^{\dagger \text { a) }}$ and Kazumasa TANAKA $^{\dagger \dagger}$, Members $^{\circ}$
}

\begin{abstract}
SUMMARY A stacked rectangular microstrip antenna with a shorting plate is proposed as a car antenna for dual band (VICS and ETC) operation in the ITS. The proposed antenna has the proper radiation patterns for the VICS and ETC. The antenna is small in size and effective in dual band operation.

key words: stacked microstrip antenna with a shorting plate, dual band operation, vehicle information and communications system (VICS), electric toll collection system (ETC)
\end{abstract}

\section{Introduction}

Recently, antennas for multi band operation have received much attention and many single feed Microstrip antennas (MSAs) for dual band operation have been proposed [1][5]. Most of them are antennas with linear polarization in two frequency bands for mobile phones and wireless LAN applications. In some ITS (intelligent transport system) applications, such as ETC (electric toll collection system), GPS (global positioning system) and SDARS (satellite digital audio radio service), a circularly polarized wave is used. Therefore, antennas for dual band operation in ITS applications must radiate a circularly polarized wave. The design of single feed and dual band MSAs with circular polarization is difficult compared with those with only linear polarization.

The authors have proposed a stacked square MSA with a shorting post as a car antenna for the vehicle information and communications system (VICS) in the ITS application [6]. Since the proposed antenna has a radiation peak at low elevation angles, the antenna receives signals with uniform level within the communication area for the VICS. Moreover, its size is much smaller than that of the conventional rectangular MSA used presently. In this letter, the antenna proposed in [6] is redesigned as a small car antenna for dual band (VICS/ETC) operation. Yuminaga et al. have proposed a single feed and triple band MSA for GPS, VICS and ETC [7]. However, the antenna hasn't been designed with consideration of the radiation patterns for VICS.

In the calculations in this letter, the simulation software package IE3D 10.2, based on the method of moments in the

Manuscript received April 20, 2007.

Manuscript revised June 8, 2007.

†The author is with the Division of System Science, Graduate School of Science and Technology, Nagasaki University, Nagasaki-shi, 852-8521 Japan.

${ }^{\dagger}$ The author is with the Department of Electrical and Electronic Engineering, Faculty of Engineering, Nagasaki University, Nagasaki-shi, 852-8521 Japan.

a)E-mail: takafumi@nagasaki-u.ac.jp

DOI: 10.1093/ietcom/e90-b.11.3307 spectral domain is used [8]. In order to ascertain the accuracy of the calculated results, the calculated return loss, axial ratio and radiation pattern are compared with experimental data.

\section{Specifications of VICS and ETC}

The communication area for the VICS extends $35 \mathrm{~m}$ in both directions from the beacon antenna installed at the shoulder of the road. In such a case, the radiation peak of the car antenna must be at low elevation angles along the road [6], [9]. The communication area for the ETC is very narrow (the extent is $4 \mathrm{~m}$ in one direction from the toll gate antenna) and the toll gate antennas are installed above the car antenna. Therefore, it is desirable that the car antenna for the ETC has a radiation peak at high elevation angles.

The center frequency $f_{0}$ and the bandwidth of the VICS in Japan are $2.4997 \mathrm{GHz}$ and $85 \mathrm{kHz}$. In order to keep high communication quality for a mobile communication system with a wide area such as VICS, it is desirable for the bandwidth to be as wide as possible. In this letter, therefore, a bandwidth of $50 \mathrm{MHz}(2.0 \%)$ is aimed for in the VICS band. The center frequency $f_{0}$ and the bandwidth of the ETC are $5.8 \mathrm{GHz}$ and $100 \mathrm{MHz}(1.73 \%)$. Linear polarization is used for the VICS while circular polarization is used for the ETC.

\section{Antenna Design}

Figure 1 shows a stacked rectangular MSA with a shorting plate and its coordinate system. The antenna consists of a dielectric substrate and an air layer with a patch. The geometry of antenna patch proposed in [6] is square. In this let-

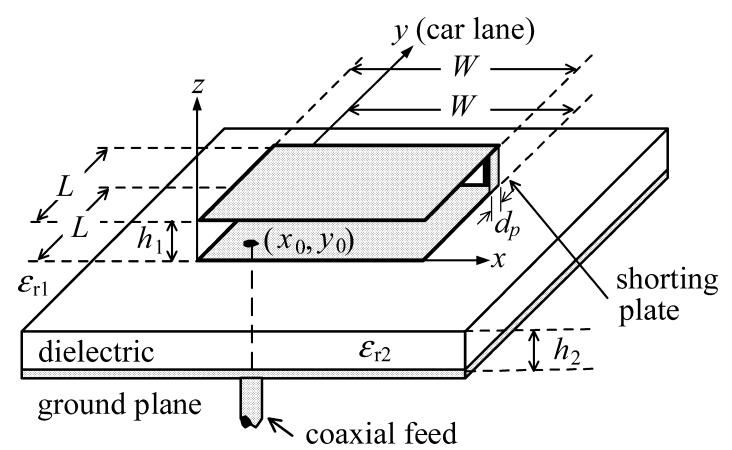

Fig. 1 Geometry of stacked rectangular microstrip antenna with a shorting plate and its coordinate system. 
ter, however, its geometry is rectangular to achieve circular polarization in the ETC band. The upper and lower rectangular patches are the same size and have a width and length of $W$ and $L$, respectively. The upper patch is shorted to the lower patch at the apex of the rectangular patch. The width of the shorting plate is $d_{p}$. The relative dielectric constant and thickness of the upper and lower layers are $\varepsilon_{r 1}=1.0, h_{1}$ and $\varepsilon_{r 2}=2.60, h_{2}$, respectively. The antenna is excited at the lower patch by a coaxial feed through the lower dielectric substrate at point $x_{0}, y_{0}$ which lays around the diagonal.

\section{Antenna Characteristics}

Figure 2 shows the difference between the frequency giving the lowest return loss and the center frequency $f_{0}=2.5 \mathrm{GHz}$ in the VICS band for changes of the width of the shorting plate $d_{p}$. Figure 2 also shows the difference between the frequency giving the best axial ratio and the center frequency $f_{0}=5.8 \mathrm{GHz}$ in the ETC band for changes of the width $d_{p}$. Although the width $d_{p}$ influences the frequency in the VICS band, it does not affect the frequency in the ETC band. In the ETC band, moreover, the return loss at the frequency giving the best axial ratio changes from $-13.5 \mathrm{~dB}$ to $-15.2 \mathrm{~dB}$, the best axial ratio from $0.34 \mathrm{~dB}$ to $0.40 \mathrm{~dB}$ and the bandwidth from $2.85 \%$ to $2.89 \%$ when the width $d_{p}$ changes from $0.8 \mathrm{~mm}$ to $2.8 \mathrm{~mm}$. The fact that the changes of the antenna characteristics in the ETC band are very small makes the designing of the antenna easier. In the design procedure, at first the size of the rectangular patch is adjusted to tune the antenna to the ETC band and after that the width of the shorting plate is adjusted to tune the antenna to the VICS band. Since independent tuning is possible for each band, the proposed antenna is effective and useful in the design for dual band operation.

Figure 3 shows the bandwidth of the antenna for changes of the thickness $h_{1}$ in the VICS and ETC bands. The bandwidth for the VICS is defined as the return loss $\leqq-10 \mathrm{~dB}$ and that for ETC as the return loss $\leqq-10 \mathrm{~dB}$ with an axial ratio $\leqq 3 \mathrm{~dB}$. For each $h_{1}$, the size of the rectangular patches and the width of the shorting plate are determined by the design procedure mentioned above and the location

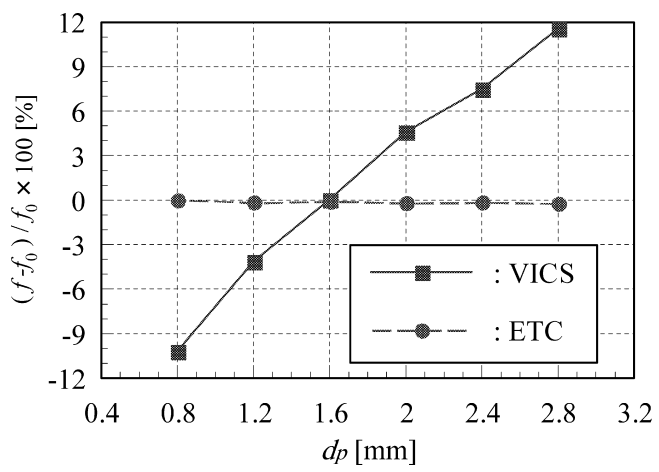

Fig. 2 Differences from center frequency $f_{0}$ of VICS and ETC ( $W=13.6 \mathrm{~mm}, L=15.1 \mathrm{~mm}, \varepsilon_{r 1}=1.0, \varepsilon_{r 2}=2.60, h_{1}=4.0 \mathrm{~mm}, h_{2}=1.6 \mathrm{~mm}$, $\left.x_{0}=3.8 \mathrm{~mm}, y_{0}=5.1 \mathrm{~mm}\right)$. of the feed point is adjusted to achieve maximum bandwidth. The bandwidths in both the VICS and ETC bands increase as the thickness $h_{1}$ increases. The bandwidths of $h_{1} \geqq 3.6 \mathrm{~mm}$ satisfy the specifications of both the VICS and ETC. For all $h_{1}$ in Fig. 3, the averaged width and length $((W+L) / 2)$ is equal to $14.35 \mathrm{~mm}=0.12 \lambda_{2.5}=0.28 \lambda_{5.8}\left(\lambda_{f}:\right.$ the wave length at $f \mathrm{GHz}$ ).

Figures 4(a) and (b) show the measured and calculated return losses and axial ratios with the thickness $h_{1}=$ $4.0 \mathrm{~mm}$, respectively. The antenna was made of copperclad Glass-fiber-PTFE. The measured bandwidth (return loss $\leqq-10 \mathrm{~dB})$ is $66 \mathrm{MHz}(2.61 \%)$ in the VICS band and the measured bandwidth (return loss $\leqq-10 \mathrm{~dB}$ with axial ratio $\leqq 3 \mathrm{~dB}$ ) is $190 \mathrm{MHz}(3.34 \%)$ in the ETC band. The bandwidths of the proposed antenna satisfy the specifica-

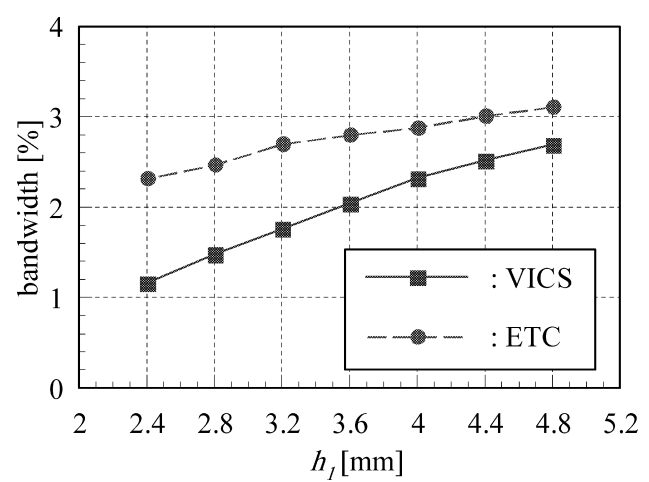

Fig. 3 Bandwidths in VICS and ETC bands $\left(\varepsilon_{r 1}=1.0, \varepsilon_{r 2}=2.60\right.$, $h_{2}=1.6 \mathrm{~mm}$ ).

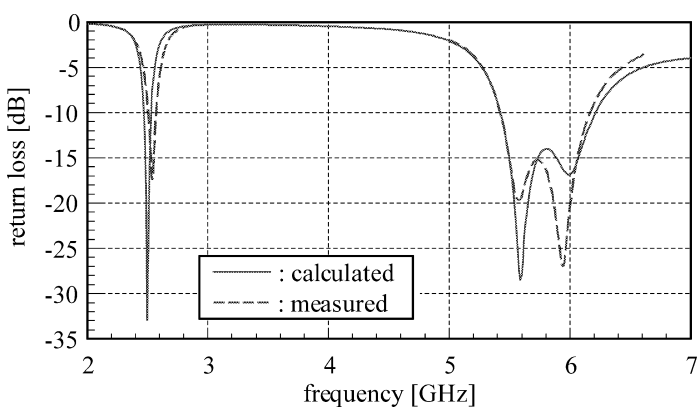

(a) Return loss.

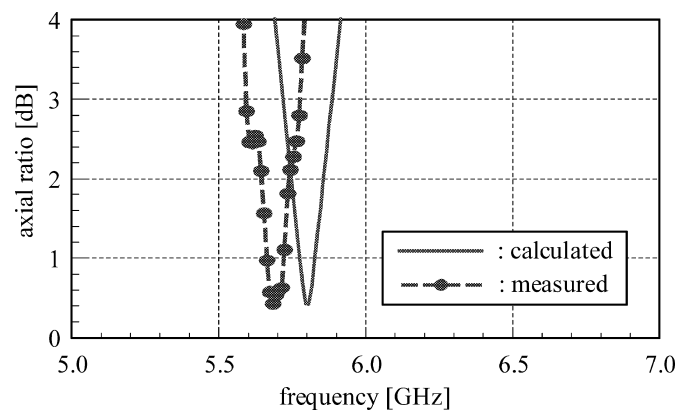

(b) Axial ratio. (in ETC band).

Fig. 4 Comparison of measured and calculated return losses and axial ratios $\left(W=13.6 \mathrm{~mm}, L=15.1 \mathrm{~mm}, d_{p}=1.6 \mathrm{~mm}, \varepsilon_{r 1}=1.0, \varepsilon_{r 2}=2.60\right.$, $\left.h_{1}=4.0 \mathrm{~mm}, h_{2}=1.6 \mathrm{~mm}, x_{0}=3.8 \mathrm{~mm}, y_{0}=5.1 \mathrm{~mm}\right)$. 


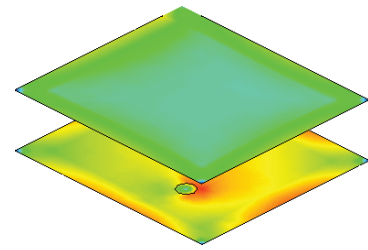

(a) Upper patch.

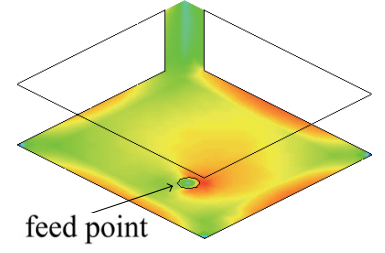

(b) Lower patch
$0 \mathrm{~dB} \quad-4 \mathrm{~dB} \quad-8 \mathrm{~dB}-12 \mathrm{~dB}-16 \mathrm{~dB}-20 \mathrm{~dB}-24 \mathrm{~dB}-28 \mathrm{~dB}-32 \mathrm{~dB}-36 \mathrm{~dB}-40 \mathrm{~dB}$

Fig.5 Time averaged electric current distributions $(W=13.6 \mathrm{~mm}$, $L=15.1 \mathrm{~mm}, d_{p}=1.6 \mathrm{~mm}, \varepsilon_{r 1}=1.0, \varepsilon_{r 2}=2.60, h_{1}=4.0 \mathrm{~mm}, h_{2}=1.6 \mathrm{~mm}$, $x_{0}=3.8 \mathrm{~mm}, y_{0}=5.1 \mathrm{~mm}$, frequency $=5.8 \mathrm{GHz}$ ).

tions of both the VICS and the ETC. The calculated results agree well with the measured ones.

Figure 5 shows the intensity of the time averaged electric current distribution at the center frequency of the ETC band, $5.8 \mathrm{GHz}$. The intensity of the electric current on most of the area of the shorting plate is very small $(-12 \mathrm{~dB}$ or less). Therefore, the shorting plate doesn't affect the antenna characteristics in the ETC band as shown in Fig. 2. The intensities of the electric currents on both the upper and lower patches (without considering the feed point) are maximum around the centers of the edges of the rectangular patches and approximately zero ( $-28 \mathrm{~dB}$ or less) around the four apexes of the patches, which indicates the width and length of the patches approximately becomes a half wavelength. However, the intensity of the electric current on the upper patch is much smaller than that on the lower patch. These characteristics of the antenna operation at $5.8 \mathrm{GHz}$ denote that the proposed antenna operates as the conventional half-wavelength stacked MSA which has the parasitic patch above the fed patch conductor [10]. Moreover, since the lower rectangular patch conductor has the feed point around the diagonal, the lower patch conductor also operates as a rectangular patch conductor with a perturbation segment in the ETC band [11]. Therefore, the proposed antenna radiates a circularly polarized wave when choosing the proper ratio $(L / W)$ of the length to the width of the rectangular patch conductor. In the conventional stacked MSA, the Quality factor $Q$ of a mutual resonance which occurs between the upper and lower patches becomes small as the thickness of the upper layer increases [12]. Therefore, as shown in Fig. 3, the bandwidth of the proposed antenna in the ETC band increases as the thickness $h_{1}$ increases.

In this letter, the electric current distribution isn't presented for the VICS band. However, the current distribution of the proposed antenna was the same as the antenna proposed in [6], which is shorted between the upper and lower square patches by a cylindrical conductor. In the VICS band, the proposed antenna resonates when the sum of the lengths of the diagonal of the upper and lower patches and the shorting plate becomes a half wavelength at $2.5 \mathrm{GHz}$. Therefore, the antenna doesn't operate as the conventional stacked MSA but as a single antenna element. In this case, therefore, as the thickness $h_{1}$ increases, the bandwidth increase

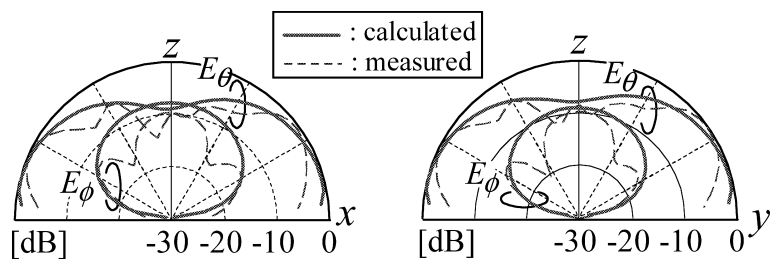

(a) $x z$ plane. $(2.5 \mathrm{GHz})$

(b) $y z$ plane. $(2.5 \mathrm{GHz})$

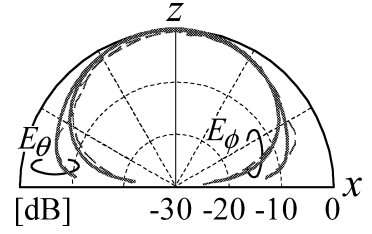

(c) $x z$ plane. $(5.8 \mathrm{GHz})$

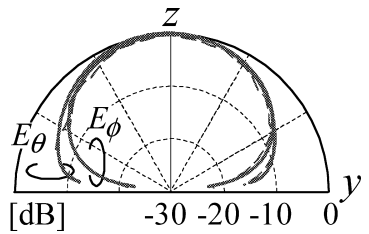

(d) $y z$ plane. $(5.8 \mathrm{GHz})$
Fig. 6 Comparison of measured and calculated radiation patterns ( $W=13.6 \mathrm{~mm}, L=15.1 \mathrm{~mm}, d_{p}=1.6 \mathrm{~mm}, \varepsilon_{r 1}=1.0, \varepsilon_{r 2}=2.60, h_{1}=4.0 \mathrm{~mm}$, $\left.h_{2}=1.6 \mathrm{~mm}, x_{0}=3.8 \mathrm{~mm}, y_{0}=5.1 \mathrm{~mm}\right)$.

(as shown in Fig. 3) is not due to a decrease in the $Q$ of the mutual resonance between the upper and lower patches, but is only a result of a decrease in the $Q$ of the upper patch.

Figures 6(a)-(d) show the measured and calculated radiation patterns at $2.5 \mathrm{GHz}$ and $5.8 \mathrm{GHz}$, respectively. Since the size of the ground plane is infinite in the calculation by IE3D, the antennas don't radiate behind the ground plane in the calculation. Moreover, the radiation pattern around $z=0$ cannot be calculated accurately. Therefore, the radiation patterns are shown for the angles $0^{\circ} \leqq \theta \leqq 85^{\circ}$. In the measurement, a finite ground plane $\left(4 \lambda_{2.5} \times 4 \lambda_{2.5} \simeq\right.$ $\left.10 \lambda_{5.8} \times 10 \lambda_{5.8}\right)$ is used. The differences between the calculated and measured radiation patterns at $2.5 \mathrm{GHz}$ are due to the edge diffractions of the finite ground plane [6]. However, the calculated radiation patterns agree well with the measured ones at $5.8 \mathrm{GHz}$ because the size of the finite ground plane is large compared with the wavelength at $5.8 \mathrm{GHz}$. At $2.5 \mathrm{GHz}$, the radiation peak is at low elevation angles which is the radiation pattern required for a car antenna for the VICS. This is due to the following: (i) the radiation fields by the electric currents on the upper and lower patches (flowing to the opposite directions) cancel out each other around the high elevation angles and (ii) the maximum radiation fields due to the electric current on the vertical shorting plate are in the broadside direction [6]. Since the proposed antenna operates as the conventional half-wavelength stacked MSA for a circular polarization at $5.8 \mathrm{GHz}$, the antenna has a radiation peak around high elevation angles where the toll gate antennas are installed.

\section{Conclusion}

A stacked rectangular MSA with a shorting plate has been proposed for dual band (VICS and ETC) operation in ITS.

The operational principles of the antenna were discussed using the electric current distributions. The return loss, axial ratio and radiation pattern were calculated by simulation software and compared with measured results. 
Although differences between the calculated and measured radiation pattern in the VICS band were observed, the good agreement between the other calculations and measurements confirm the results of this work.

The proposed antenna is very small and has suitable radiation patterns for the VICS and ETC. Moreover, since independent tuning is possible for each band, the proposed antenna is effective and useful in the design for dual band operation.

\section{Acknowledgments}

The return loss, the axial ratio and the radiation pattern of the MSA were measured at the Joint Research and Development Center, Saga University. The authors would like to thank Assistant Professor E. Nishiyama of Saga University for his valuable advice on the measurement of MSA. This research was supported in part by a Grant-in-Aid for Scientific Research (16760305) from Japan Society for the Promotion of Science.

\section{References}

[1] K.L. Wong, "Compact dual-frequency and dual-polarized microstrip antennas," in Compact and Broadband Microstrip Antennas, ed. K. Chang, pp.87-161, John Wiley \& Sons, New York, 2002.

[2] K.L. Wong, "Broadband dual-frequency and dual-polarized microstrip antennas," in Compact and Broadband Microstrip Antennas, ed. K. Chang, pp.279-293, John Wiley \& Sons, New York, 2002.

[3] Y.X. Guo, K.M. Luk, and K.F. Lee, "A quarter-wave U-shaped patch antenna with two unequal arms for wideband and dual-frequency operation," IEEE Trans. Antennas Propag., vol.50, no.8, pp.10821087, Aug. 2002

[4] J.H. Lu, "Broadband dual-frequency operation of circular patch antennas and arrays with a pair of L-shaped slots," IEEE Trans. Antennas Propag., vol.51, no.5, pp.1018-1023, May 2003.

[5] S.H. Yeh, K.L. Wong, T.W. Chiou, and S.T. Fang, "Dual-band planar inverted $\mathrm{F}$ antenna for GSM/DCS mobile phones," IEEE Trans. Antennas Propag., vol.51, no.5, pp.1124-1126, May 2003.

[6] T. Fujimoto, S. Noguchi, K. Tanaka, and M. Taguchi, "Stacked square microstrip antenna with a shorting post for road vehicle communication," RF and Microwave Computer-Aided Engineering, vol.14, no.3, pp.244-252, May 2004.

[7] S. Yuminaga and Y. Yamada, "A triple-layer patch antenna capable of triple-frequency operation," 2003 IEEE Antennas and Propagation Society International Symposium, in CD, 2003.

[8] IE3D User's Manual, Zeland Software, Inc., Dec. 1999

[9] Y. Kazama, N. Morita, and S. Tokumaru, "Mobile communications higher-order-mode microstrip antennas for road vehicle communications," IEICE Trans. Commun. (Japanese Edition), vol.J82-B, no.10, pp.1898-1904, Oct. 1999.

[10] A.N. Tulintseff, S.M. Ali, and J.A. Kong, "Input impedance of a probe-fed stacked circular microstrip antenna," IEEE Trans. Antennas Propag., vol.39, no.3, pp.381-390, March 1991.

[11] K.R. Carver and J.W. Mink, "Microstrip antenna technology," IEEE Trans. Antennas Propag., vol.29, no.1, pp.2-24, Jan. 1981.

[12] S.D. Targonski, R.B. Waterhouse, and D.M. Pozar, "Design of wideband aperture-stacked patch microstrip antennas," IEEE Trans. Antennas Propag., vol.46, no.9, pp.1245-1251, Sept. 1998. 